STOUT, E.L.

Math. Zeitschr. 95, 403-404 (1967)

\title{
Corrections to
}

\section{On Some Algebras of Analytic Functions on Finite Riemann Surfaces}

\author{
Math. Zeitschr. 92, 366-379 (1966) \\ E.L. Stout
}

Eingegangen am 5. Oktober (1966)

Page 371 , line 10 from the bottom of the page. The definition of $F_{j}$ should $\operatorname{read} F_{j}\left(\zeta_{1}, \ldots, \zeta_{N}\right)=h_{j}\left(\zeta_{1}\right)$

Page 372 , line 10 from the top of the page. The statement "... if $F$ is in $H_{p}\left[U^{N}\right]$, then $F \circ H$ is in $H_{p}[R]$ "' is erroneous as has been pointed out to me by Prof. W. RuDIN who has communicated the following fact. Let $H[U]$ be the space of functions holomorphic in $U$, the open unit disc, and let

$$
\mathfrak{B}=\left\{g \in H[U]: \iint_{U}|g(x+i y)|^{2} d x d y<\infty\right\} .
$$

Theorem. If for $f \in H_{2}\left[U^{2}\right]$ we define $f_{D} \in H[U]$ by $f_{D}(z)=f(z, z)$, then the map $f \rightarrow f_{D}$ carries $H_{2}\left[U^{2}\right]$ onto $\mathfrak{B}$.

Remark. Since $\mathfrak{B}$ is much larger than $\mathrm{H}_{2}[U]$, this result shows that the quoted statement is in error.

Proof. If

then

$$
f(z, w)=\sum_{m, n \geqq 0} c_{m n} z^{m} w^{n} \text { and } f_{D}(z)=\sum_{k \geqq 0} \gamma_{k} z^{k}
$$

so

$$
\gamma_{k}=\sum_{m+n=k} c_{m n}
$$

Thus,

$$
\left|\gamma_{k}\right|^{2} \leqq(k+1) \sum_{m+n=k}\left|c_{m n}\right|^{2}
$$

$$
\frac{1}{\pi} \iint_{U}\left|f_{D}\right|^{2}=\sum_{k \geqq 0} \frac{\left|\gamma_{k}\right|^{2}}{k+1} \leqq \sum_{m, n \geqq 0}\left|c_{m n}\right|^{2}=\|f\|_{2}^{2},
$$

and consequently if $f \in H_{2}\left[U^{2}\right], f_{D} \in \mathfrak{B}$.

On the other hand, if $g \in \mathfrak{B}$,

$$
g(z)=\sum_{k \geqq 0} \gamma_{k} z^{k}
$$


put

$$
f(z, w)=\sum_{m, n \geqq 0} \frac{\gamma_{m+n}}{1+m+n} z^{m} w^{n}
$$

Then $f \in H_{2}\left[U^{2}\right]$ and $g=f_{D}$. Thus, the map $f \rightarrow f_{D}$ is onto, and we have the result.

Apparently open is the problem of characterizing the functions $f_{D}$ for $f \in H_{p}\left[U^{2}\right], p \neq 2, \infty$.

In spite of the incorrect assertion contained in its proof, Theorem II. 3 of the paper is correct as stated, and the indicated proof is sufficient. The proof suggested requires only a very special- and correct-case of the assertion, viz, that in which $F$ is of the form $F\left(\zeta_{1}, \ldots, \zeta_{N}\right)=h\left(\zeta_{1}\right)$ for $h \in H_{p}[U]$. For such a choice of $F, F \circ H$ is indeed in $H_{p}[R]$ : If $u$ is a harmonic majorant of $F$, then $u \circ \eta_{1}$ is a harmonic majorant for $F \circ H$ on $R$. The proof is now concluded as before.

Yale University, New Haven, Connecticut, U.S.A. 